JoHn C. HILL and F. K. WOHN

Ames Laboratory, Iowa State University, Ames, Iowa, U.S.A. A. WOLF", 2. BEPANT", and R.L. GIII Brookhaven National Laboratory, Upton, N.Y., U.S.A. H. KRUSE ${ }^{+}$

Michlgan State Univeraity, E. Lansing, Mich., U.S.A.

Facilities at the TRISTAN mass separator for measurement of nagnetic moments of nuclear excited states are described and recent results for the $N=82$ isotones are presented.

NOTISE Pentious of thIS REPORT ARE ILEEAR! R. It has been reproduced from the $\cdots \cdot \cdot$ t avallable copy to permil the broaciosit. 1. Introduction possible availablity.

Measurement of the static magnetic dipole momente of nuclear excited states are of interest since they reveal information on nuclear structure not avallable by other means. A Eystem has been constructed at the TRISTAN separator to measure magnetic dipole moments of excited states in neutron-rich nuclei using the method of perturbed angular correlations (PAC). High magnetic fields are now available through the use of a superconducting magnet. The capability of the TRISTAN system will be discussed and the PAC measuring apparatus will be described. Final results from recent $g$ factor measurements at TRISTAN on $4^{+}$states in the $N=82$ isotones will be discussed in some detail. Studies in progress will be briefly outlined.

\title{
2. The TRISTAN separator
}

TRISTAN is a mass separator on-line to the High Flux Beam Reactor (HFBR) at Brookhaven National Laboratory which is used for studies of the structure of neutron-rich nuclei far from stability. Nuclei of interest are produced by fission of ${ }^{235} \mathrm{U}$ by thermal neutrons in a target ion-source combination located in a neutron flux of $2 \times 10^{10}$ part/s in an external beam line at the HFBR.

The layout of the TRISTAN system is shown below in Fig. 1. The fission products emerging from the ion source are mass separated by a $90^{\circ}$ magnet and subsequently switched by a second magnet to the appropriate experimental station. All measurements of $g$ factors both with the normal and superconducting magnets have been carried out at the $45^{\circ}$ port as indicated in Fig. 1 .

A large number of g-factor measurements are possible at TRISTAN due to the great variety of elements available for mass separation and the long life of the available ion sources. This situation was brought about by a vigorous program of ion source development. The first ion sources were developed by Shmid et al.[1] and used surface ionization on Ta or Re surfaces at temperatures greater than $2000^{\circ} \mathrm{C}$. The Ta ionizer is desirable for experiments on the alkalis $\mathrm{Rb}$ and Cs. The Re ionizer is optimum for the production of

"Present address: Physics Department, Nuclear Research Centre, Beer Sheva, Israel.

+Present address: Felco Research Corporation, 1818 Division street, Nashville, Tenn., U.S.A. 


\section{DISCLAIMER}

This report was prepared as an account of work sponsored by an agency of the United States Government. Neither the United States Government nor any agency thereof, nor any of their employees, makes any warranty, express or implied, or assumes any legal liability or responsibility for the accuracy, completeness, or usefulness of any information, apparatus, product, or process disclosed, or represents that its use would not infringe privately owned rights. Reference herein to any specific commercial product, process, or service by trade name, trademark, manufacturer, or otherwise does not necessarily constitute or imply its endorsement, recommendation, or favoring by the United States Government or any agency thereof. The views and opinions of author expressed herein do not necessarily state or reflect those of the United States Government or any agency thereof. 
beams of Sr, Ba, Ce, and Pr. A long-lived (to 1500 hours) FBBIAD ion source modeled after that of Kirchner[2] is used to produce beams of $\mathrm{Cu}, \mathrm{Zn}, \mathrm{Ga}, \mathrm{Ge}$, Se, Br, $R r, R b, A g, C d, I i n, S n, S b, T e, I$, Xe, and Cs.

A second generation of high temperature ion sources has been recently developed which operate up to temperatures of $2500^{\circ} \mathrm{C}$ allowing fast release times for short-lived fission products and study of nuclei very far from stabllity. The thermal ion source described by Piotrowski et al. [3] produces beams of Ga, Ge, As, Rb, isr, Y, In, Sn, Cs, Ba, La, Ce, Pr, Nd, Pm, Sm, Eu, $\mathrm{Gd}, \mathrm{Tb}, \mathrm{Dy}$, and Ho. Also a high temperature plasma ion source produces beams of Cu, Zn, Ga, Ge, As, Se, Br, Kr, Rb, Sr, Y, Ag, Cd, In, Sn, Sb, Te, I, Xe, Cs, Ba, Ce, and Pr.

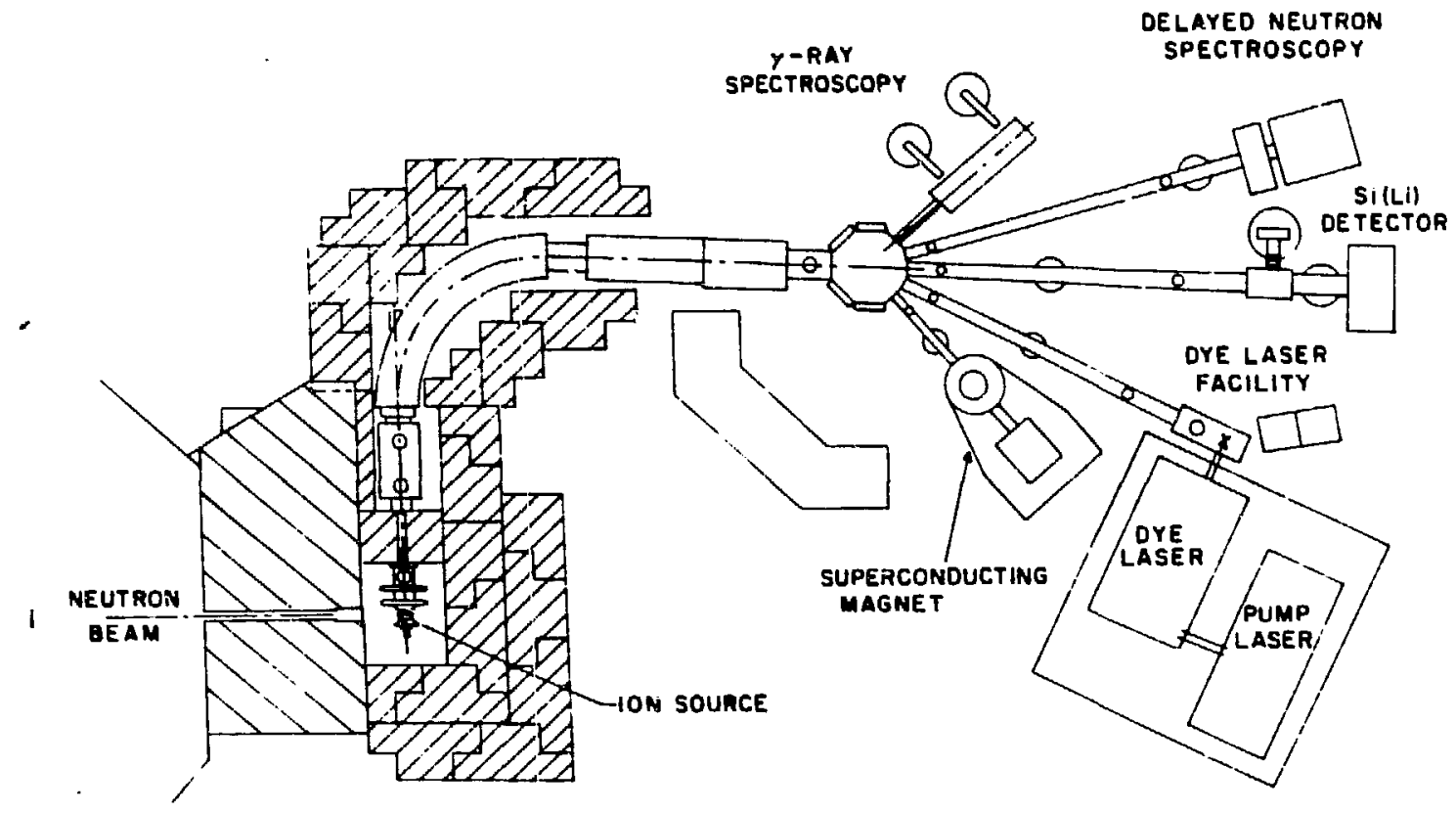

TRISTAN

Pig. 1. Layout of TRISTAN separator system

3. The PAC measurement syst:em

In order to determine magnetic moments of nuclear excited states by the PAC method it is necessary to observe the precession of the angular correlation between two $Y$ rays in a static magnetic field. Two basic approaches have been used. [4] If the half-life of the state of interest is long conpared to the time resolution of the electronic system the Larmor frequency is measured directly by measuring the precession of the angular correlation in a static magnetic field (differential PAC). Such measurements can be made on states with half-lives longer than approximately $10 \mathrm{~ns}$ and require sufficiently large angular correlation coefficients $A_{22}, A_{44}$, but it is not necessary to know their exact values.

If the half-life of the state of interest is shorter than the time resolution of the electronic system, the integral PAC method must be used. In order to extract the $g$ factor, values for the state half-life and the coefficients $\mathrm{A}_{22}, \mathrm{~A}_{44}$ must be avallable. Also one must determine the attenuation (if any) of the correlation. 
In -factor measurements at TRIsTAN the experimental aystem consists of up to four atationary Ge detectors with efficiencies of 17 to 20 placed at about $8 \mathrm{~cm}$ from the center of the pole pieces as shown in lig. 2 . In such a configuration it is possible to measure the correlation at six different angles simultaneously. The electronic and data acquisition systens are set in such a way that $\gamma-\gamma$ coincidences between any of the six pairs of detectors are recorded as address triplets on magnetic tape. The four-detector system has been described in detail by wolf et al.[5]

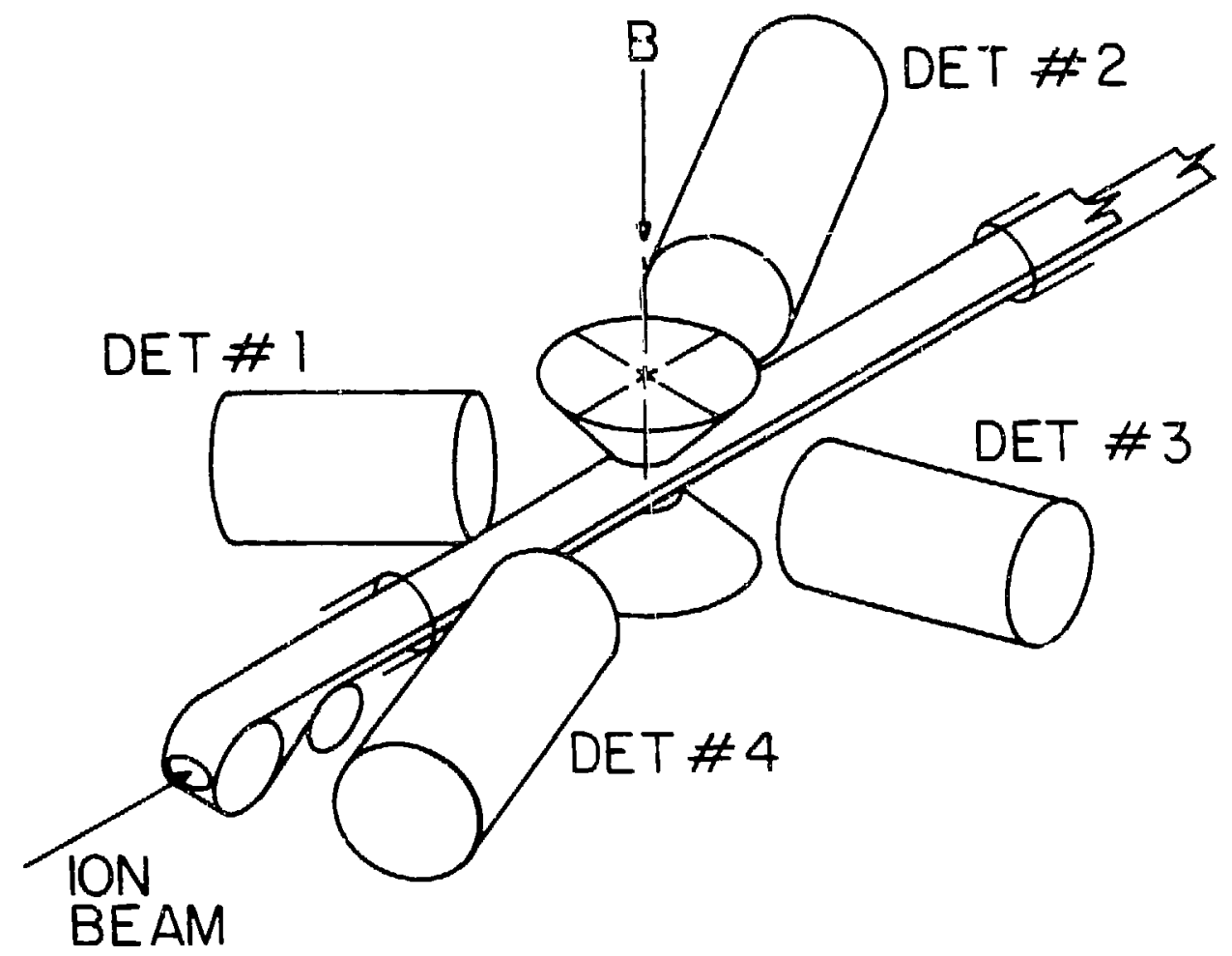

Fig. 2. Schematic view of four detector system, magnet, and tape collector used in PAC measurements.

In order to determine the $g$ factor two different experiments are carried out: a) an unperturbed angular correlation to determine the coefficients $\mathrm{A}_{22}{ }^{\prime \prime}$ $A_{44}$ in the Legendre polynomial expansion; b) a perturbed angular correlation in a static magnetic field. A source $1 \mathrm{~cm}$ in diameter was collected on an aluminized mylar tape in the TRISTAN beam line and moved to a position in the static magnetic field with transport times ranging from 0.3 to $0.6 \mathrm{~s}$. In the first round of experiments a maynetic field of up to $2.6 \mathrm{~T}$ was provided by a conventional electromagnet.

The magnetic field at the site of the nucleus of interest was taken to be the applied magnetic field. Corrections to this field due to the $\mathrm{knight}$ shift or dlamagnetic shielding are negligible and perturbations from electric field gradients ase not expected due to the cubic structure of aluminum. In addition unperturbed correlations on a number of $\gamma$-ray cascades were measured using the same type of aluminized mylar tape and good agreement with theoretical values was oltained, $[5,6]$ 
Experimentally we measured the double ratio:

$$
R(\theta)=\left[\frac{I(\theta, B)}{I(\theta,-B)} / \frac{I(-\theta, B)}{I(-\theta,-B)}\right]^{1 / 2}
$$

where $I(\theta, B)$ is the number of counts for the given $\gamma-\gamma$ cascade at angle $\theta$ with manetic field $B$ up. The advantage of using this ratio is that it does not depend on normalization involving detector efficiency, geometry, beam intensity, and counting time with field up and down. Several sources of systematic errors are thus eliminated. For a given $\gamma$ - $\gamma$ correlation, $R(\theta)$ has a maximum at an angle $\theta_{\max }$ which depends on $A_{22}$ and $A_{44^{\circ}}$

\section{The superconducting magnet for PAC studies}

A superconducting magnet system was installed and successfully tested in the Spring of 1984 and the first measurements were undertaken in June 1984. The system consists of a split pole Nb-Ti superconducting magnet housed in a ${ }^{4}$ He cryostat which contains a $\lambda$-point refrigeration unit. The maximum field avallable is $6.25 \mathrm{~T}$ at $4.2{ }^{\circ} \mathrm{K}$ and $6.75 \mathrm{~T}$ if the $\lambda$-point refrigerator is used to cool the magnet to $2.2^{\circ} \mathrm{K}$. The above values constitute a factor of three incresse in strength over those avallable at TRISTAN using conventional electromagnets. The magnetic fleld is homogeneous to better than 18 over the $1 \mathrm{~cm}^{2}$ area typical of our sources. The activity is collected as before on an aluminized mylar tape and woved to the measuring point in the magnetic field through a room temperature horizontal bore tube through the center of the magnet. A maximum of four Ge detectors can be positioned in the plane perpendicular to the fisld direction at a minimum distance of $8.5 \mathrm{~cm}$ from the source. The superconducting magnet system was designed such that absorbing material beitiaen source and the Ge detectors is minimized.

A schematic side view of the magnet and its housing is shown in Fig. 3 . The cryostat has a holding time for the liquid 4 He of 3 to 4 days and field reversal requires a total time of 3 to 4 hours. At the top of Fig. 3 a surve showing the radial dependence of the magnetic field inside the bore tube is given. The curve at the left shows the magnitude and vertical deperdence of the fringing field just outside the housing.

The possibility of measuring a given g factor by the integral PAC method depends on the size of the coefficients $A_{22}$ and $A_{44}$ and the value of the halflife of the measured state. Since the Larmor precession frequency is directly proportional to the magnetic field, the increase of three in $B$ obtained with the superconductor allows one to measure $g$ factors for states with shorter half-lives or to use $\gamma$-ray cascades with smaller $A_{22}$ or $A_{44}$ coefficients, thus eniarging considerably the number of possible measurements. Another important factor is that experiments can be done with a given statistical accuracy in about 10 of the time needed with the old (nonsuperconducting) system.

\section{5. g-factor measurements at TRISTAN}

A number of $g$-factor measurements have been carried out at TRISTAN using both the differential and integral pAC methodg. The $g$ factors for the $2{ }^{+}$

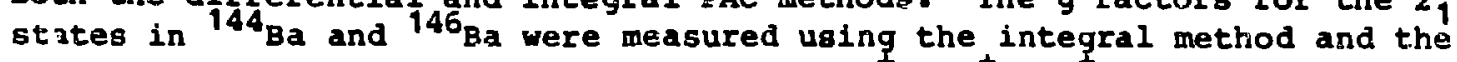
normal magnet. The cascades measured were $\mathrm{o}_{2}^{+}+2_{1}^{+}+0_{1}^{+}$which are particularly advantageous due to the large anisotropy of the above spin sequence. The results were interpreted within the framework of the IBA-2 formalism, and confirmed the validity of assuming a drastic change in the proton boson number when the number of neutrons is increased from 88 to 90 . This assumption allows the results to be explained in a simple framework involving constant 
values of boson $g$ factors. This work has been discussed in detail elsawhere. [7]

The differential PAC technique has been used to masure[8] the $g$ factor of the $102 \mathrm{~ns} 7 / 2^{+}$state in $97 \mathrm{zr}$ at $1264 \mathrm{keV}$. The value of $0.39 \pm 0.04$ is connistent with the simple shell model prediction, $g($ Schmidt) $=+0.43$, astuming $g_{s}=g_{s}$ (free). Recently measurements have been made on the $6{ }_{1}^{+}$state $\left(T_{1 / 2}=0.14 \mathrm{\mu s}\right)$ at $1774 \mathrm{keV}$ in 132 Te using the differential PAC method. Integral PAC measurements on the $2_{1}{ }^{+}$states in ${ }^{146} \mathrm{Ce}$ and ${ }^{148} \mathrm{Ce}$ are in progress using the ouperconducting anget.

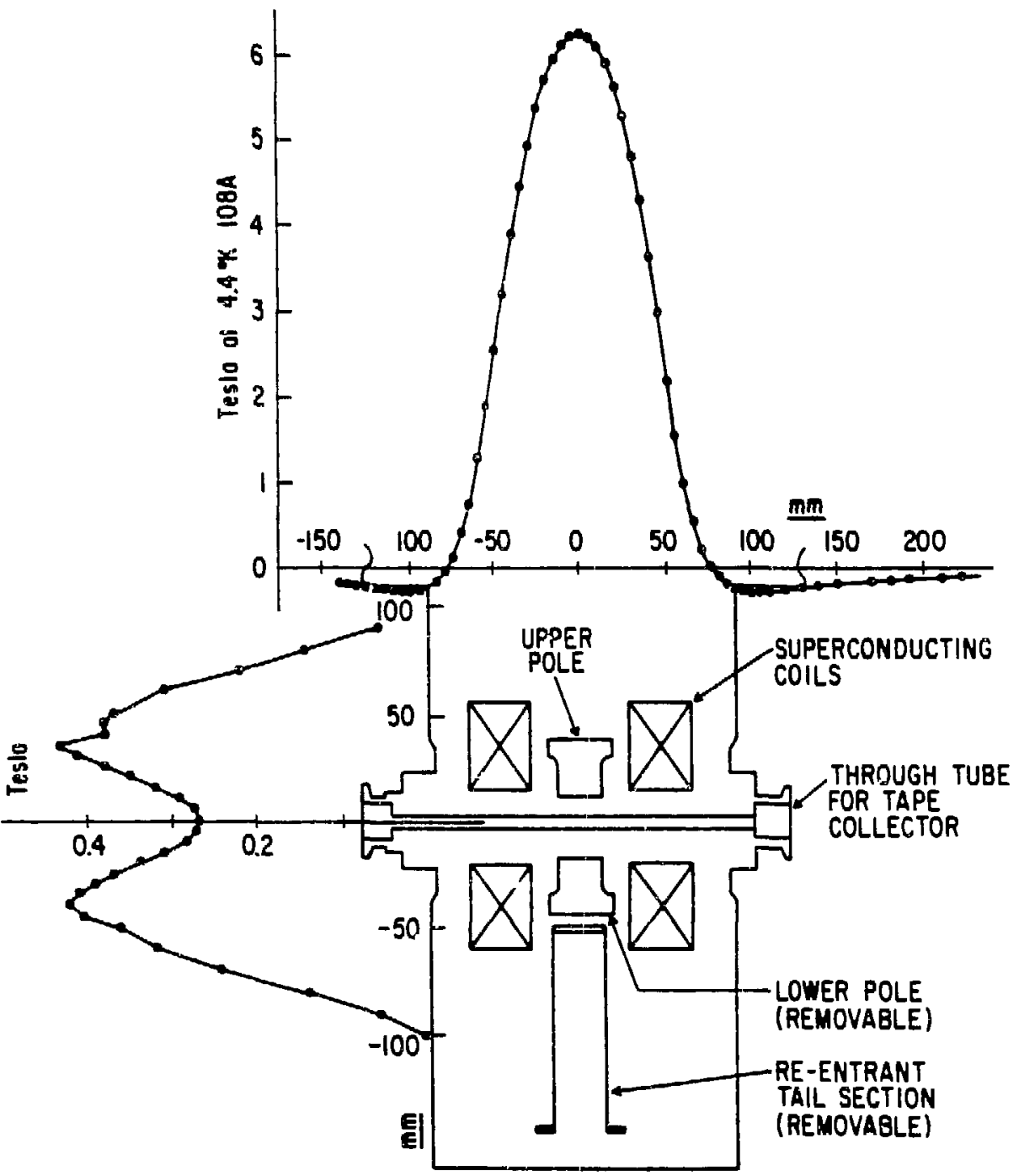

Fig. 3. Schematic Bide view of the TRISTAN superconducting magnet. For explanation of curves see text.

6. g factors of $4_{1}^{+}$states in the $N=82$ isotones

The $\mathrm{N}=82$ isotones have been extensively studied both experimentally and theoretically: The existing experimental data is quite comprehensive with the exception of otatic magnetic dipole and electric quadrupole moments. In particular, the only known $g$ factor for $2^{+}$or $4_{1}$ atates in these nuclei is $\mathrm{g}\left(4_{1}{ }^{+}\right)$for ${ }^{140} \mathrm{Ce}$. The values measured here for $4_{1}{ }^{+}$states in $136 \mathrm{xe}$ and ${ }^{138} 8_{\mathrm{Ba}}$ enable 18 to establish systematics for $g\left(4_{1}^{+}\right)$, for the $N=82$ isotones and provide a test of shell-model calculations. 
6.1. Experimental determination of $g\left(4,{ }^{+}\right)$for ${ }^{136}$ xe and ${ }^{139} \mathrm{Ba}$

The $g$ factors were measured by the integral PAC method as described above using normal magnet. The beame of ${ }^{136} \mathrm{I}$ and ${ }^{138} \mathrm{Cs}$ parents were supplied by the FEBIAD type ion cource of the gRISTAN eystem. The magnetic fleld was 17.2 and $18.3 \mathrm{~kg}$ for the $X e$ and Ba measurements respectlvely. The half-lives of the $4_{1}^{+}$states in $136 \times$ and $138_{\mathrm{Ba}}$ are known to be 1.32 and $2.17 \mathrm{~ns}$, respectively $[9,10]$. The experimental system consisted of four Ge detectors placed about $8 \mathrm{~cm}$ from the center of the pole pieces (see Fig. 2 ).

An unperturbed angular correlation measurement was first carried out to determine the Iegendre coefficients $\lambda_{22}$ and $A_{44}$ followed by an integral PAC measurement to determine the $g$ factor. For the PAC measurements the detectors were set so that between 4 pairs we had $130^{\circ}$ (for $136 \mathrm{xe}$ ) and $140^{\circ}$ (for $138_{\mathrm{Ba}}$. The angles were chosen to maximize $\mathrm{R}(\theta)$ for the respective nuclei. we thus had four times more statistics than possible with a two-detector ayatem.

Partial decay schemes of ${ }^{136} \mathrm{Xe}$ (Ref. [11]) and 138 $\mathrm{Ba}$ (Ref. [12]) are given in Fig. 4. We measured unperturbed $\gamma-\gamma$ correlations for some of the cascades in Fig. 4. In Fig. 5 some of the measured correlations are presented. The solid lines are best fits to a Legendro polynomial expansion up to and $\frac{1}{3}$ ncluding $P_{4}(\cos \theta)$. We assign $\left(3^{+}, 5^{+}\right)$to the $J^{h}$ of the $2444-k e v$ level in $136 \mathrm{Xe}$ inçe the corfelqtion of the $750-381-\mathrm{keV}$ cascade is consistent with both the $3^{+}-4^{+}-2^{+}$or $5^{+}-4^{+}-2^{+}$spin sequences. The 750-381-kev cascade in 136 xe and the $409-463-\mathrm{keV}$ cascade in $138_{\mathrm{Ba}}$ have large $\mathrm{A}_{22}$ coefficients and are thus suitable for PAC measurements. Also the skip cascades $705-(381)-1313$ in ${ }^{136} x$ and $409-(463)-1463$ in ${ }^{138}$ Ba were used to improve the statistics. The PAC measurements were carried out as described above.
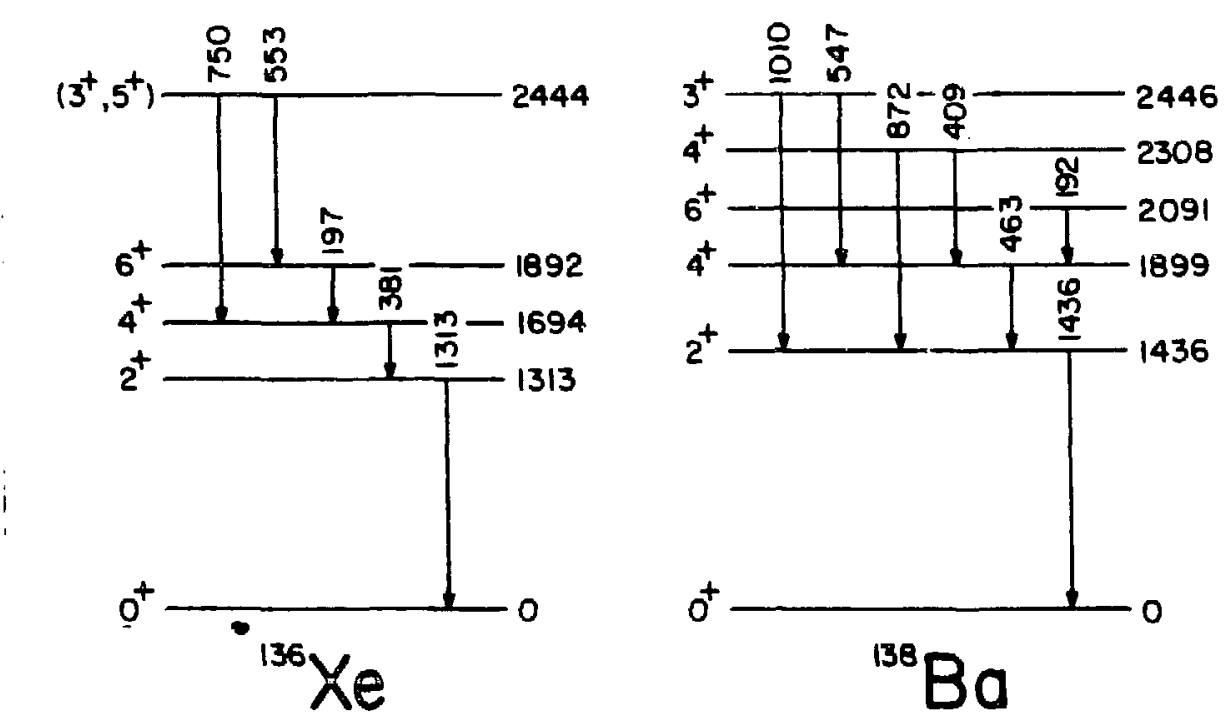

Fig. 4. Partial decay schemes for ${ }^{136} \mathrm{xe}$ and ${ }^{138_{\mathrm{Ba}}}$ relevant to the g-factor meagurements.

Results of the measurements of $R(\theta)$ for various cascaden in ${ }^{136} \mathrm{Xe}$ and

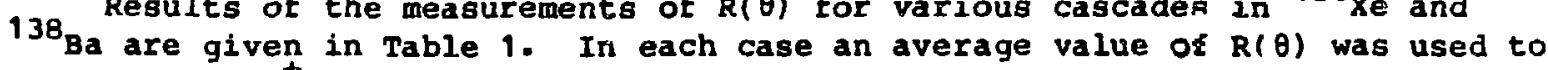
calculate $g\left(4_{1}{ }^{+}\right)$. The values are given in Table 1 . 
6.2. Shell-model calculations for $g\left(4_{1}^{+}\right)$in the $N=82$ isotones.

Shel 1 -model calculations were carried out for $g\left(4_{1}{ }^{+}\right)$in the $\mathrm{N}=82$ isotones ${ }^{134} \mathrm{Te},{ }^{136} \mathrm{Xe}, 138 \mathrm{Ba}$, and ${ }^{140} \mathrm{Ce}$. The appropriate wavefunctions were determined using a modified surface delta interaction (MSDI) residual interaction. [13] The model space consisted of all $g_{7 / 2}, d_{5 / 2}, h_{11 / 2}, d_{3 / 2}$ ' and $\$_{1 / 2}$ configurations outside of a closed ${ }^{132} \mathrm{sn}$ core. The only restriction was that a maximum of four protons were allowed in the $h_{11 / 2}$ orbit and that only configurations with overall seniority less than or equal to four were considered. values for the individual matrix elements of the two-body Hamiltonian and for the singie particle energies were fitted using a set of 150 well established experimental energy levels for the $N=82$ isotones.

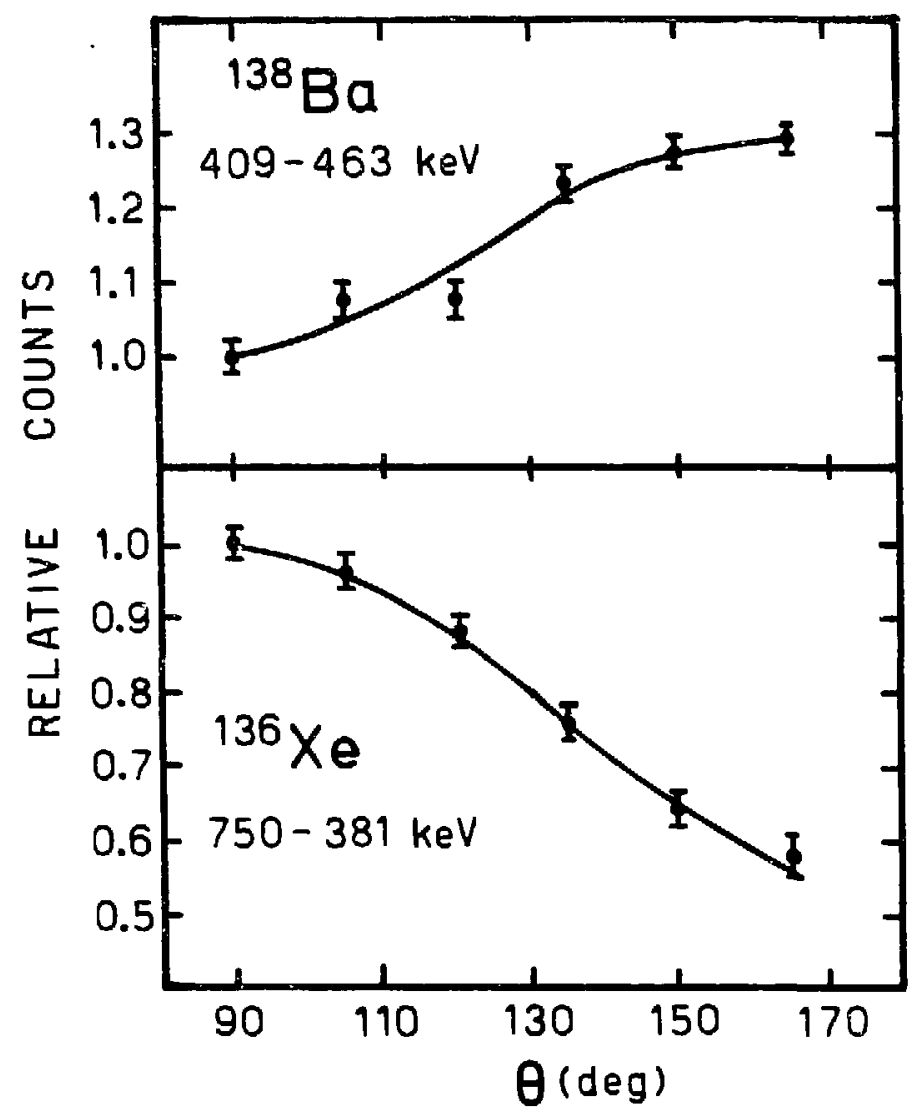

Fig: 5. Results of angular correlation measurements for the $4^{+}-4^{+}-2^{+}$cascade in $138_{B a}$ and the $\left(3^{+}, 5^{+}\right)-4^{+}-2^{+}$cascade in $136^{X e .}$

It is well known that due to core polarization effects $[14,15], \frac{1}{3} \frac{1}{4}$ is not possible to describe scae $g$ factors in this region [i.e. $g\left(6, i_{1}\right.$ in ${ }^{134}$ Te] using configuration-mixed shell-model wavefunctions alone. We have determined a set of effective proton $g$ factors $g_{l}^{\prime}$ and $g_{g}^{\prime}$ by fitting the ground state $g$ factors of ${ }^{139} \mathrm{La}$ and $14{ }_{\mathrm{Pr}}$ to the configuration mixed wavefunctions described above. Fit: to the experimental values $y^{i}$ ld $g_{l}^{\prime}=1.12$ and $g_{g}^{\prime}=4.12$. The effective proton $g$ factors were subsequently used to calculate g factors for all $g\left(4_{1}{ }^{7}\right)$. The results are given in Table 2 .

The experimental $\mathrm{g}\left(4_{1}{ }^{+}\right.$) for ${ }^{136} \mathrm{Xe},{ }^{138_{\mathrm{Ba}}} \mathrm{\text {,and }} 1{ }^{140} \mathrm{Ce}$ agree within experimental error with calculations using the configuration-mixed shell-model wavefunctions described above as can be seen from Table 2 . It thus appears that a single set of effective proton $g$ factors can adequately describe the core polarization effects for all three nuclei. In both $136_{\mathrm{Xe}}$ and $138_{\mathrm{Ba}}$ more 
then 75 of the contribution to the ${ }_{136}$ factor comes from a ingle conponent of

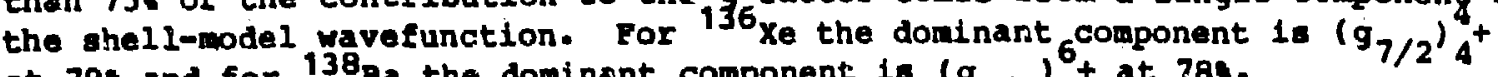
at 798 and for $138 \mathrm{Ba}$ the dominant component $18\left(9_{7 / 2}\right)^{6}+$ at 784 .

\section{TABLE 1.}

Regults of angular correlation and PAC mesarements

\begin{tabular}{|c|c|c|c|}
\hline pucleus & $\begin{array}{l}\text { Cascade enargies (keV) } \\
\text { Spin sequence }\end{array}$ & $\mathbf{R}(\theta)$ & $g\left(4_{1}{ }^{+}\right)$ \\
\hline \multirow[t]{5}{*}{$136 \times e$} & $750-381$ & $0.868 \pm 0.033$ & \\
\hline & $(3,5)^{+}-4^{+}-2^{+}$ & & \\
\hline & & & $0.80 \pm 0.15$ \\
\hline & $750-(381)-1313$ & $0.844 \pm 0.030$ & \\
\hline & $(3,5)^{+}-4^{+}-2^{+}-0^{+}$ & & \\
\hline \multirow[t]{5}{*}{$138_{\mathrm{Ba}}$} & $409-463$ & $1.094 \pm 0.010$ & \\
\hline & $4^{+}-4^{+}-2^{+}$ & & \\
\hline & & & $0.80 \pm 0.14$ \\
\hline & $409-(463)-1436$ & $1.097 \pm 0.013$ & \\
\hline & $4^{+}-4^{+}-2^{+}-0^{+}$ & & \\
\hline
\end{tabular}

Table 2.

Calculated and measured $g$ factors for $4_{1}^{+}$states in the $N=82$ isotones

\begin{tabular}{|c|c|c|c|}
\hline \multirow[t]{2}{*}{ Nucleus } & \multirow{2}{*}{ g(experimental) } & \multicolumn{2}{|c|}{ g(calculated) } \\
\hline & & Free proton g factor & Effective proton $g$ factor ${ }^{a}$ \\
\hline $\mid \begin{array}{l}134 \mathrm{re} \\
136_{\mathrm{Xe}} \\
138 \mathrm{Ba} \\
140 \mathrm{Ce}\end{array}$ & $\begin{array}{l}0.80 \pm 0.15^{b} \\
0.80 \pm 0.15^{b} \\
1.11 \pm 0.04^{c}\end{array}$ & $\begin{array}{l}0.57 \\
0.58 \\
0.67 \\
0.99\end{array}$ & $\begin{array}{l}0.84 \\
0.84 \\
0.90 \\
1.11\end{array}$ \\
\hline
\end{tabular}

$g_{\ell}^{\prime}=1.12$ and $g_{s}^{\prime}=4.12$

bThis work

CRef. [16]

\section{Conelusion}

A prograw to measure magnetic dipole moments of nuclear excited states is now established at the TRISTAN separator. In order to extend these measurements to states with shorter half-lives and to low-yleld isotopes, a superconducting magnet has been 1 ngtalled and $g$ factor measurements are in progress. Measurements of $\mathrm{g}\left(4_{1}^{+}\right)$for the $\mathrm{N}=82$ isotones ${ }^{136} \mathrm{Xe}$ and $138_{\mathrm{Ba}}$ indicate that the reaults can be described by calculations employing configurationmixed shell-rodel wavefunctions and a single set of effective proton $g$ factors that account in an empirical fashion for core polarization effects. 
We acknowladge the use of complter programs written by Alex Brown in our calculation of the magnetic momente. The shell-model wavefunctions were calculated by a group led by $\mathrm{B}_{n} \mathrm{H}$. Wildenthal. This study was supported by the U.S. Department of Energy, Offlce of High Energy and Nuclear Physics, under Contracts No. H-7405-eng-82 at Ames and DE-AC02-76CH0016 at Brookhaven.

\section{REFEREMCES}

[1] M. Shmid, R. I. Gill, and C. Chung, Nucl. Instr. and Meth. 211, 287 (1983).

[2] R. Kirchner, Nucl. Ingt, and Meth. 186, 275 (1981).

[3] A. Piotrowski, R. I. Gill, and D. C. McDonald, Nucl. Instr. and Meth. (in press).

[4] H. Frauenfelder and R. M. Steffen, In alpha-, beta-, and gamma-ray spectroscopy, ed. K. Siegbahn, vol. 2 (North-Holland, Ansterdam, 1965), P. 1151.

[5] A. Wolf, C. Chung, w. B. Walters, G. Peaslee, R. L. Gill, M. Shmid, V. Manzella, E. Meler, M. L. Stelts: H. I. Liou, R. E. Chrien, and D. S. Brenner, Nucl. Instr. Heth. 206,397 (1983).

[6] A. WolE, C. Chung. W. B. Walters, R. L. Gill, H. Shmid, R. E. Chrien, and G. Peaslee, Phys. Rev. C $\underline{28}, 352$ (1983).

[7] A. Wolf, Z. Berant, D. D. Warner, R. I. Gill, M. Shmid, R. E. Chrien, G. Peaslee, H. Yamanoto, J. C. Hill, F. K. Wohn, C. Chung, and W. B. walters, Phys. Lett. 123B, 165 (1983).

[8] Z. Berant, R. I. Gill, M. H. Rafailovich, R. E. Chrien, J. C. Hill, F. K. Wohn, R. F. Petry, C. Chung, G. Peaslee, and M. Mohsen, BAPS 2B, 701 (1983).

[9] K. Kawade, G. Battistuzxi, H. Lawin, K. Sistemich, and J. Blomquist, Z. Physik A298, 187 (1980).

[10] W. M. Currie, Nucl. Phys. 48, 561 (1963).

[11] W. R. Western, J. C. Hill, W. L. Talbert, Jr., and W. C. Schick, Jr., Phys. Rev. C 15, 1822 (1977).

[12] L. K. Peker, Hucl. Data Sheets 36, 289 (1982).

[13] P. W. M. Glaudemans, P. J. Brussaard, and B. H. Wildenthal, Nucl. Phys. A102, $593(1967)$.

[14] A. Wolf and E. Cheifetz, Phys. Rev. Iett. 36, 1072 (1976).

[15] K. Heyde, M. Waroquier, P. Van Isacker, and H. Vincx, Phys. Rev. C 16, 489 (1977).

[16] I. K. Peker, Nucl. Data Sheets 28, 267 (1979). 